\title{
ON COMPUTER-ASSISTED PROVING THE EXISTENCE OF PERIODIC AND BOUNDED ORBITS
}

\author{
ROMAN SRZEDNICKI
}

Annual Lecture dedicated to the memory of Professor Andrzej Lasota

\begin{abstract}
We announce a new result on determining the Conley index of the Poincaré map for a time-periodic non-autonomous ordinary differential equation. The index is computed using some singular cycles related to an index pair of a small-step discretization of the equation. We indicate how the result can be applied to computer-assisted proofs of the existence of bounded and periodic solutions. We provide also some comments on computer-assisted proving in dynamics.
\end{abstract}

\section{Remarks on the history of computer assisted proofs}

\subsection{Computer assistance in mathematics}

The assistance of computers in developing mathematics is probably as old as computers themselves. Computers are applied in numerical simulations leading to formulating and testing new theorems or in rigorous proving. The latter applications consist, in particular, in complete formalizations of proofs

Received: 01.03.2015.

(2010) Mathematics Subject Classification: 37B30, 37C27, 65L99.

Key words and phrases: Poincaré map, Conley index, interval arithmetic, rigorous numerical algorithm.

Supported by the EU under the Toposys project FP7-ICT-318493-STREP. 
(like the system Mizar initiated by Andrzej Trybulec in 1973, c.f. [32]), in proving complicated formulas (requiring, for example, long symbolic calculations with Maple or Mathematica packages), or in providing rigorous assessments using interval arithmetic (c.f. [17, 24]). Relying in computers within rigorous mathematics is sometimes contested; due to possible errors in the processor or in the program code, one cannot be sure whether a computer performs exactly the same calculations as one expects (recall the Pentium processor bug discovered in 1994, c.f. [44]).

It seems the four-color theorem was the first famous result which was proved with the aid of a computer (in 1976 by Kenneth Appel and Wolfgang Haken; c.f. 1, 2]; compare also [41] for a simpler proof). Other important results proved with the computer assistance include the solution of the Kepler conjecture about dense sphere packing, announced in 1998 (c.f. [12]) and completed only recently in [13], and the proof of the non-existence of finite projective planes of order 10 (c.f. [21, 22]). It should be noted that sometimes computer-assisted proofs of important results predated the 'classical' proofs; that was, for example, in the case of the solution of the Robbins problem in Boolean algebras (c.f. [27] and [26]) and of the isoperimetric problem on two pieces of spheres separated by a flat disc (the 'double-bubble conjecture', c.f. [14] and [18]).

\subsection{Computer assisted proofs in dynamical systems}

Perhaps the first essential result related to dynamics, proved with the computer assistance, was the solution of the Feigenbaum conjecture given in 1982 (c.f. [23, 24]). It refers to the existence of a solution of a certain functional equation arising in period-doubling bifurcations of iterations of unimodular maps. A related important result obtained at that time with the computer's aid was given in [8]. Computer-assisted proofs of results on the Lorenz system of equations

$$
\dot{x}=\sigma(y-x), \quad \dot{y}=\rho x-x z-y, \quad \rho z=x y-\beta z,
$$

where $\sigma, \rho$, and $\beta$ are scalar parameters, started to appear in the 90 s of the last century. The numerical evidence of a chaotic behavior of the system with $\sigma=10, \rho=28$, and $\beta=\frac{8}{3}$ was announced in 1963 by Edward Lorenz in 25]. Since then, hundreds of papers devoted to explanation of the dynamics of the system were published. In [15, 16, the shooting method based on the intermediate value property was applied in order to prove results on homoclinic orbits of (1). The first rigorous result which explained the chaotic nature of (1) was announced by Konstantin Mischaikow and Marian Mrozek in [28] in 1995 and was explained in details in the consecutive papers [29, 30]. 
Proofs of all basic result related to the Lorenz system, predicted by numerical evidence, were given by Warwick Tucker at the turn of the century (c.f. [42, 43]). The publication of papers mentioned above inspired research on the computer-assisted proofs in dynamics. In particular, Hubbard's and Saari's conjectures in mechanics were proved in, respectively, [3] and [33]. At present several groups of mathematicians specializes in that kind of research; among them there are cooperating teams CAPA (Computer-aided proofs in analysis) at Uppsala, CAPD (Computer assisted proofs in dynamics) at Kraków, and CHomP (Computational homology project) at Rutgers University; see the corresponding web-pages [4, 5, 7]. The achievements of CAPD include results on problems of celestial mechanics (e.g. [6, 19, 20, 47]), on KuramotoSivashinsky equations (e.g. [31, 40, 45, 51]), and on complicated behavior of Hénon, Rössler, and other dynamical systems (e.g. 9, 10, 46, 48, 49, 50]).

Up to now the vast majority of proofs of essential results on the system (1) and similar ones, are computer-assisted. The difficulty in 'classical' proving qualitative results for those systems lies mainly in the lack of analytic formulas for solutions; the required estimates are given numerically and the errors of these estimates grow exponentially as time goes to infinity. Usually it is impossible to perform all such calculations 'by hand' and therefore an aid of computers is necessary.

Computer assisted proofs in dynamical systems are frequently based on topological tools being far reaching improvements of the intermediate value property. They include the Brouwer degree (on which the method of covering relations introduced by Piotr Zgliczyński is based, c.f. [11]), the fixed point index, and the Conley index. The papers [35, 36] explain how the latter tool can be applied. In the remaining part of the present text we describe a recent research on that subject.

\section{On the Conley index of Poincaré maps}

\subsection{Dynamical systems}

We begin with a recollection of some basic definitions of the dynamical systems theory. Let $\mathbb{T}$ denote either the set of real numbers $\mathbb{R}$ or the set of integer numbers $\mathbb{Z}$. Let $X$ be a topological space. A continuous map $\phi: X \times$ $\mathbb{T} \rightarrow X$ is called a dynamical system on $X$ if it satisfies

$$
\begin{aligned}
& \forall x \in X: \phi(x, 0)=x, \\
& \forall x \in X, s, t \in \mathbb{T}: \phi(x, s+t)=\phi(\phi(x, s), t) .
\end{aligned}
$$


The set $\mathbb{T}$ represents time. We call $X$ the phase space of $\phi$ and we denote by $\phi_{t}$ the map $x \mapsto \phi(x, t)$. For $x \in X$ the set $\left\{\phi_{t}(x): t \in \mathbb{T}\right\}$ is called the orbit of $x$. An orbit is called bounded provided its closure is compact. If an orbit consists of exactly one point, it is called stationary. A non-stationary orbit is called periodic if $\phi_{T}(x)=x$ for some $T>0$ (such a $T$ is called a period). Clearly, each periodic orbit is bounded. The problem of existence of periodic orbits belongs to the most important ones in the theory of dynamical systems. It is frequently solved by topological methods applied to the estimates obtained by numerical approximations, like in several computer-assisted results on chaotic behavior of the Lorenz and Rössler systems, mentioned in Subsection 1.2. In fact, chaotic dynamics is often indicated by the existence of an infinite set of periodic orbits.

Let $A \subset X$. The invariant part of $A$ (with respect to $\phi$ ) is defined as

$$
\operatorname{Inv}(A):=\left\{x \in A: \phi_{t}(x) \in A \forall t \in \mathbb{T}\right\} .
$$

A set $A \subset X$ is called invariant provided $A=\operatorname{Inv}(A)$. A compact invariant set $S$ is called isolated if it is maximal invariant set in some its neighborhood.

In the case $\mathbb{T}=\mathbb{Z}, \phi$ is called a discrete-time dynamical system; the properties (2) and (3) imply $\phi$ can be regarded as the family of iterations of the homeomorphism $\phi_{1}$. On the other hand, if $g: X \rightarrow X$ is a homeomorphism then the formula $\phi^{g}(x, n):=g^{n}(x)$ for $x \in X$ and $n \in \mathbb{Z}$ defines a discrete-time dynamical system, i.e. $\phi^{g}$ is the family of iterations of $g$ (hence $\phi_{1}^{g}=g$ ). By abuse of terminology, we frequently write $g$ to mean the dynamical system $\phi^{g}$.

If $\mathbb{T}=\mathbb{R}, \phi$ is called a continuous-time dynamical system. Usually such a system is generated by the solutions of an autonomous ordinary differential equation

$$
\dot{x}=v(x),
$$

where $v: \mathbb{R}^{n} \rightarrow \mathbb{R}^{n}$ is a smooth vector-field. Actually, not every autonomous equation generates a dynamical system since, in general, solutions are not defined in the whole $\mathbb{R}$. That problem can be easily overcome by a suitable modification of $v$, if we are interested in qualitative properties of solutions in a bounded region of the phase space. Here we deal with bounded orbits mainly, hence in the sequel we assume all considered equations generate dynamical systems.

\subsection{Discrete-time Conley index}

Assume $X$ is a metrizable locally compact space. Let $g: X \rightarrow X$ be a homeomorphism and let $N$ and $L$ be compact subsets of $X, L \subset N$. The pair 
$(N, L)$ is called a weak index pair (for $g$ ) if

$$
\begin{aligned}
& S:=\operatorname{Inv}(\overline{N \backslash L}) \subset \operatorname{int}(N \backslash L), \\
& g(L) \cap N \subset L, \\
& \overline{g(N) \backslash N} \cap N \subset L .
\end{aligned}
$$

It follows $S$ is isolated invariant set; $(N, L)$ is therefore also called a weak index pair for $S$. (A more restrictive notion of index pair is not used in the present text.) It follows by (5) and (6) the map

$$
g_{(N, L)}: N / L \rightarrow N / L, \quad g_{(N, L)}(x):= \begin{cases}g(x), & \text { if } x, g(x) \in N \backslash L, \\ *, & \text { otherwise }\end{cases}
$$

is continuous; it is called the index map. (Here $N / L$ denotes $(N \backslash L) \cup\{*\}$ endowed with the quotient topology, where $*$ is a point outside of $N$.) Let $H$ denote the singular homology functor with coefficients in the field of rational numbers $\mathbb{Q}$. The index map induces a graded endomorphism

$$
H\left(g_{(N, L)}\right): H(N / L, *) \rightarrow H(N / L, *) .
$$

Denote by $R \phi$ the Leray reduction of an endomorphism $\phi: V \rightarrow V$ of a vector space $V$ over $\mathbb{Q}$. It is defined as follows. Put

$$
K V:=V / \bigcup_{k} \operatorname{ker} \phi^{k}
$$

the quotient of $V$ by the generalized kernel of $\phi$. Let $K \phi: K V \rightarrow K V$ be the induced monomorphism. If $V$ is finite dimensional, $R \phi:=K \phi$. In the general case put

$$
R V:=\bigcap_{k} \operatorname{Im}(K \phi)^{k}
$$

and define $R \phi: R V \rightarrow R V$ as the restriction of $K \phi . R \phi$ is thus an automorphism of $R V$. In an obvious way one extends the definition of $R$ to the graded endomorphisms.

The Conley index of $S$ (denoted $\mathrm{CH}(S, g)$ ) is defined as the conjugacy class of the graded automorphism $R H\left(g_{(N, L)}\right)$. It follows by [34, 37], each isolated invariant set $S$ has at least one week index pair $(N, L)$ satisfying (4) and the definition is independent of the choice of $(N, L)$. The name of the index commemorates Charles Conley (1933-1984), who invented an analogous 
tool for continuous-time dynamical systems in the 70 s of the 20th century, improving the retract method of Tadeusz Ważewski.

The basic property of the index is the following one: if $\mathrm{CH}(S, g) \neq 0$ then $S$ is nonempty; in particuar there exists a bounded orbit. Moreover, if $X$ is an ENR (Euclidean neighborhood retract; for example $\mathbb{R}^{n}$, a manifold, a polyhedron or a cubical set) and the Lefschetz number $\Lambda(\mathrm{CH}(S, g))$ is nonzero then $g$ has a fixed point. Recall that the Lefschetz number of a graded endomorphism $\psi:=\left\{\psi_{n}\right\}_{n}$ of finite dimensional spaces, such that $\psi_{n}=0$ for almost all $n$, is defined as

$$
\Lambda(\psi):=\sum_{n=0}^{\infty}(-1)^{n} \operatorname{trace} \psi_{n} .
$$

Actually, the Conley index has other properties; in particular it is additive and multiplicative (in some sense), hence it provides an 'algebraic' information on the 'size' of the isolated invariant set, in a slightly similar way like the fixed point index provides an information on the number of fixed points of a map.

\subsection{The Poincaré map}

Roughly speaking, a Poincaré map is the first-return map to a section of a dynamical system. Usually, after a change of variables such a map can be reduced to the translation operator of a time-periodic non-autonomous equation. Below we state the formal definition of that reduced case only.

Let $T>0$ and let $f: \mathbb{R} \times \mathbb{R}^{n} \rightarrow \mathbb{R}^{n}$ be a smooth and bounded timedependent vector-field such that the map $t \mapsto f(t, x)$ is $T$-periodic for each $x$. The system of equations

$$
\dot{t}=1, \quad \dot{x}=f(t, x)
$$

generates two dynamical systems:

$$
\begin{aligned}
& \phi \text { on } \mathbb{R} / T \mathbb{Z} \times \mathbb{R}^{n}, \\
& \psi \text { on } \mathbb{R} \times \mathbb{R}^{n} .
\end{aligned}
$$

The Poincaré map $P: \mathbb{R}^{n} \rightarrow \mathbb{R}^{n}$ is defined for $x \in \mathbb{R}^{n}$ by the formula

$$
\psi_{T}(0, x)=(T, P(x)) .
$$

It follows, in particular, the dynamical system $\phi$ has a $T$-periodic orbit if and only if $P$ has a fixed point. 


\subsection{A theorem on the Conley index of the Poincaré map}

We follow the notation from subsection 2.3 . Moreover, for $s \in \mathbb{R} / T \mathbb{Z}$ and a set $Z \subset \mathbb{R} / T \mathbb{Z} \times \mathbb{R}^{n}$ we define the $s$-section of $Z$ as

$$
Z_{s}:=\left\{x \in \mathbb{R}^{n}:(s, x) \in Z\right\} .
$$

Assume $h>0$ and assume $(N, L)$ is a weak index pair for $\phi_{h}$. Let $S$ be equal to the invariant part of $\overline{N \backslash L}$ with respect to $\phi_{h}$. One can prove that $S$ is also an isolated invariant set for the continuous-time system $\phi$, and $S_{0}$, the 0 -section of $S$, is an isolated invariant set for $P$. Consequently, if $\mathrm{CH}\left(S_{0}, P\right) \neq 0$ (hence $S_{0}$ is nonempty) then $\phi$ has a bounded orbit and if $\Lambda\left(\mathrm{CH}\left(\mathrm{S}_{0}, \mathrm{P}\right)\right) \neq 0$ (hence $S_{0}$ contains a fixed point of $P$ ) then $\phi$ has a $T$-periodic orbit. Therefore our aim is to find a method of determining the Conley index of $S_{0}$.

By $(\widetilde{N}, \widetilde{L})$ we denote the lift of $(N, L)$ to $\mathbb{R} \times \mathbb{R}^{n}$, i.e.

$$
\begin{aligned}
& \widetilde{N}:=\left\{(t, x) \in \mathbb{R} \times \mathbb{R}^{n}:(t \bmod T, x) \in N\right\}, \\
& \widetilde{L}:=\left\{(t, x) \in \mathbb{R} \times \mathbb{R}^{n}:(t \bmod T, x) \in L\right\} .
\end{aligned}
$$

Let $u$ and $v$ be singular cycles on $\left(N_{0}, L_{0}\right) .(u, v)$ is called a pair of contiguous cycles over $[0, T]$ provided there exist singular chains $w$ on $\widetilde{N} \cap\left([0, T] \times \mathbb{R}^{n}\right)$ and $z$ on $\widetilde{L} \cap\left([0, T] \times \mathbb{R}^{n}\right)$ such that

$$
\partial w=0 \times u-T \times v+z,
$$

where $0 \times u$ and $T \times v$ denote the images of the cycles $u$ and $v$ under the chain maps induced by the embeddings $x \mapsto(0, x)$ and, respectively, $x \mapsto(T, x)$.

By $|c|$ we denote the support of a $q$-dimensional singular cycle $c=\sum_{i} \alpha_{i} \sigma_{i}$, i.e.

$$
|c|=\bigcup_{i} \sigma\left(\Delta_{q}\right)
$$

where $\Delta_{q}$ is the standard $q$-dimensional simplex. The pair of contiguous cycles $(u, v)$ is called $h$-movable if

$$
\psi_{t}(|w|) \subset \widetilde{N}, \quad \psi_{t}(|z|) \subset \widetilde{L}
$$

for each $t \in[0, h]$. 
Theorem 1 (compare [39, Theorem 4.7]). Assume $\frac{T}{h} \in \mathbb{Q}$ and both $N_{0}$ and $L_{0}$ be ENRs. Let $k:=\operatorname{dim} H\left(N_{0}, L_{0}\right)$, let $A=\left[a_{i j}\right]$ be a graded $(k \times k)$-matrix over $\mathbb{Q}$, and let

$$
\left(u_{j}, \sum_{i=1}^{k} a_{i j} u_{i}\right), \quad j=1, \ldots, k,
$$

be h-movable pairs of contiguous cycles over $[0, T]$ such that the homology classes of $u_{j}$ form a basis of $H\left(N_{0}, L_{0}\right)$. Then the Conley index $\mathrm{CH}\left(S_{0}, P\right)$ is equal to the conjugacy class of $R A$.

A proof of Theorem 1 can be found in [39]. A similar theorem, less practical in applications, appeared already in [38]. As a consequence of Theorem 1 one gets the following result.

Corollary 1. Under assumptions of Theorem 1, if $R A \neq 0$ then $\phi$ has a bounded orbit and if $\Lambda(A) \neq 0$ then $\phi$ has a T-periodic orbit.

A simple case of Theorem 1 implies another corollary.

Corollary 2. If $N_{0}$ and $L_{0}$ are ENRs, $H\left(N_{0}, L_{0}\right)$ is 1-dimensional and there exists a pair of $h$-movable cycles $(u, v)$ such that the homology class of $u$ is a generator of $H\left(N_{0}, L_{0}\right)$, and

$$
v=u \quad \text { or } \quad v=-u
$$

then the equation $\dot{x}=f(t, x)$ has a T-periodic solution.

\subsection{On application of Theorem 1 to computer-assisted proving}

As it was already pointed out, usually it is difficult (or even impossible, due to limitations of computers) to find a satisfactory approximation of the map $\phi_{T}$ if $T$ is large. On the other hand, for relatively small values of $h$, the $h$-discretization $\phi_{h}$ can be computed with a good precision and its essential properties can be rigorously proved. Contrary to the other computer-assisted results on Poincaré maps, Theorem 1 allows to avoid any computations related to the values of solutions at times greater then $h$.

Theorem 1 requires an index pair of ENRs for $\phi_{h}$. For a given differential equation, such a pair consisting of cubical sets, can be found by programs taken from the libraries of CAPD (accessible in the web-page [5]). Let $(N, L)$ be such a pair. For a given basis $\left[u_{j}\right]$ of homology classes in $H\left(N_{0}, L_{0}\right)$, one should push forward each cycle $u_{j}$ in order to obtain corresponding chains 
$w$ and $z$, and the cycle $v_{j}$. Up to some technical details that is enough: the homology classes $\left[v_{j}\right]$ are represented in the basis by some matrix $A$ and Theorem 1 asserts the Conley index is equal to the conjugacy class of the Leray reduction of $A$. As it was indicated in Corollary 1, the reduction provides an information on the existence of bounded and periodic orbits. Based on Theorem 1, an algorithm for computation of the index for some planar equation is constructed in [39].

\section{References}

[1] Appel K., Haken W., Every planar map is four colorable. Part I: Discharging, Illinois J. Math. 21 (1977), 429-490.

[2] Appel K., Haken W., Koch J., Every planar map is four colorable. Part II: Reducibility, Illinois J. Math. 21 (1977), 491-567.

[3] Bánhelyi B., Csendes T., Garay B.M., Hatvani L., A computer-assisted proof of $\Sigma_{3}$ chaos in the forced damped pendulum equation, SIAM J. Appl. Dyn. Syst. 7 (2008), 843-867.

[4] CAPA, http://www2.math.uu.se/ warwick/CAPA

[5] CAPD, http://capd.ii.uj.edu.pl

[6] Capiński M.J.,Computer assisted existence proofs of Lyapunov orbits at L2 and transversal intersections of invariant manifolds in the Jupiter-Sun PCR3BP, SIAM J. Appl. Dyn. Syst. 11 (2012), 1723-1753.

[7] CHomP, http://chomp.rutgers.edu

[8] Eckmann J.-P., Koch H., Wittwer P., A computer-assisted proof of universality for area-preserving maps, Mem. Amer. Math. Soc. 47 (1984), 1-121.

[9] Galias Z., Computer assisted proof of chaos in the Muthuswamy-Chua memristor circuit, Nonlinear Theory Appl. IEICE 5 (2014), 309-319.

[10] Galias Z., Tucker W., Numerical study of coexisting attractors for the Hénon map, Int. J. Bifurcation Chaos 23 (2013), no. 7, 1330025, 18 pp.

[11] Gidea M., Zgliczyński P., Covering relations for multidimensional dynamical systems, J. Differential Equations 202 (2004), 33-58.

[12] Hales T.C., A proof of the Kepler conjecture, Ann. of Math. (2) 162 (2005), 1065-1185.

[13] Hales T.C. et al., A formal proof of the Kepler conjecture, preprint (2015), http:// arxiv.org/pdf/1501.02155.pdf

[14] Hass J., Schlafly R., Double bubbles minimize, Ann. of Math. (2) 151 (2000), 459-515.

[15] Hassard B., Zhang J., Existence of a homoclinic orbit of the Lorenz system by precise shooting, SIAM J. Math. Anal. 25 (1994), 179-196.

[16] Hastings S.P., Troy W.C., A shooting approach to the Lorenz equations, Bull. Amer. Math. Soc. 27 (1992), 128-131.

[17] Hickey T., Ju Q., van Emden M.H., Interval arithmetic: From principles to implementation, J. ACM 48 (2001), 1038-1068.

[18] Hutchings M., Morgan F., Ritoré M., Ros A., Proof of the double bubble conjecture, Ann. of Math. (2) 155 (2002), 459-489.

[19] Kapela T., Simó C., Computer assisted proofs for non-symmetric planar choreographies and for stability of the Eight, Nonlinearity 20 (2007), 1241-1255.

[20] Kapela T., Zgliczyński P., The existence of simple choreographies for the N-body problem - a computer assisted proof, Nonlinearity 16 (2003), 1899-1918. 
[21] Lam C.W.H., The search for a finite projective plane of order 10, Amer. Math. Monthly 98 (1991), 305-318.

[22] Lam C.W.H., Thiel L., Swiercz S., The non-existence of finite projective planes of order 10, Canad. J. Math. 41 (1989), 1117-1123.

[23] Lanford O.E., III, A computer-assisted proof of the Feigenbaum conjecture, Bull. Amer. Math. Soc. (N.S.) 6 (1982), 427-434.

[24] Lanford O.E., III, Computer-assisted proofs in analysis, in: Proceedings of the International Congress of Mathematicians, Berkeley, California, USA, 1986, pp. 1385-1394.

[25] Lorenz E.N., Deterministic nonperiodic flow, J. Atmos. Sci. 20 (1963), 130-141.

[26] Mann A.L., A complete proof of the Robbins conjecture, preprint (2003).

[27] McCune W., Solution of the Robbins problem, J. Autom. Reasoning 19 (1997), 263276.

[28] Mischaikow K., Mrozek M., Chaos in the Lorenz equations: A computer assisted proof, Bull. Amer. Math. Soc. (N.S.) 32 (1995), 66-72.

[29] Mischaikow K., Mrozek M., Chaos in the Lorenz equations: A computer assisted proof. II: Details, Math. Comp. 67 (1998), 1023-1046.

[30] Mischaikow K., Mrozek M., Szymczak A., Chaos in the Lorenz equations: A computer assisted proof. III: Classical parameter values, J. Differential Equations 169 (2001), $17-56$.

[31] Mischaikow K., Zgliczyński P., Rigorous numerics for partial differential equations: the Kuramoto-Sivashinsky equation, Found. Comput. Math. 1 (2001), 255-288.

[32] Mizar project, http://mizar.org

[33] Moeckel R., A computer-assisted proof of Saari's conjecture for the planar three-body problem, Trans. Amer. Math. Soc. 357 (2005), 3105-3117.

[34] Mrozek M., Leray functor and cohomological Conley index for discrete dynamical systems, Trans. Amer. Math. Soc. 318 (1990),149-178.

[35] Mrozek M., From the theorem of Wazewski to computer assisted proofs in dynamics, Banach Center Publ. 34 (1995), 105-120.

[36] Mrozek M., Topological invariants, multivalued maps and computer assisted proofs in dynamics, Comput. Math. Appl. 32 (1996), 83-104.

[37] Mrozek M., Index pairs algorithms, Found. Comput. Math. 6 (2006), 457-493.

[38] Mrozek M., Srzednicki R., Topological approach to rigorous numerics of chaotic dynamical systems with strong expansion, Found. Comput. Math. 10 (2010), 191-220.

[39] Mrozek M., Srzednicki R., Weilandt F., A topological approach to the algorithmic computation of the Conley index for Poincaré maps, SIAM J. Appl. Dyn. Syst. 14 (2015), $1348-1386$.

[40] Mrozek M., Żelawski M., Heteroclinic connections in the Kuramoto-Sivashinsky equations, Reliab. Comput. 3 (1997), 277-285.

[41] Robertson N., Sanders D., Seymour P., Thomas R., The four-colour theorem, J. Combin. Theory Ser. B 70 (1997), 2-44.

[42] Tucker W., The Lorenz attractor exists, C.R. Math. Acad. Sci. Paris 328 (1999), 11971202.

[43] Tucker W., A rigorous ODE solver and Smale's 14 th problem, Found. Comput. Math. 2 (2002), 53-117.

[44] Wikipedia, Pentium FDIV bug, http://en.wikipedia.org/wiki/Pentium FDIV bug

[45] Wilczak D., Chaos in the Kuramoto-Sivashinsky equations - a computer assistē proof, J. Differential Equations 194 (2003), 433-459.

[46] Wilczak D., The existence of Shilnikov homoclinic orbits in the Michelson system: a computer assisted proof, Found. Comput. Math. 6 (2006), 495-535.

[47] Wilczak D., Zgliczyński P., Heteroclinic connections between periodic orbits in planar restricted circular three body problem - a computer assisted proof, Comm. Math. Phys. 234 (2003), 37-75.

[48] Wilczak D., Zgliczyński P., Period doubling in the Rössler system - a computer assisted proof, Found. Comput. Math. 9 (2009), 611-649. 
[49] Wilczak D., Zgliczyński P., Computer assisted proof of the existence of homoclinic tangency for the Hénon map and for the forced-damped pendulum, SIAM J. Appl. Dyn. Syst. 8 (2009), 1632-1663.

[50] Zgliczyński P., Computer assisted proof of chaos in the Rössler equations and in the Hénon map, Nonlinearity 10 (1997), 243-252.

[51] Zgliczyński P., Rigorous numerics for dissipative partial differential equations II. Periodic orbit for the Kuramoto-Sivashinsky PDE - a computer assisted proof, Found. Comput. Math. 4 (2004), 157-185.

Institute of Mathematics

Faculty of Mathematics and Computer Science

JAGIELLONIAN UNIVERSITY

LOJASIEWICZA 6

30-348 KraKóW

POLAND

e-mail: srzednicki@im.uj.edu.pl 